\title{
Parametric modeling and control of telescope wind-induced vibration
}

Douglas G. MacMynowski, George Z. Angeli, Konstantinos Vogiatzis, Joeleff Fitzsimmons, Stephen Padin

Douglas G. MacMynowski, George Z. Angeli, Konstantinos Vogiatzis, Joeleff Fitzsimmons, Stephen Padin, "Parametric modeling and control of telescope wind-induced vibration," Proc. SPIE 5497, Modeling and Systems Engineering for Astronomy, (16 September 2004); doi: 10.1117/12.552282

SPIE Event: SPIE Astronomical Telescopes + Instrumentation, 2004, Glasgow, United Kingdom 


\title{
Parametric modeling and control of telescope wind-induced vibration
}

\author{
Douglas G. MacMynowski ${ }^{a}$, George Z. Angeli ${ }^{b}$, Konstantinos Vogiatzis $^{b}$, \\ Joeleff Fitzsimmons ${ }^{c}$ and Steve Padin ${ }^{d}$ \\ ${ }^{a}$ California Institute of Technology \\ Department of Control and Dynamical Systems, Pasadena CA 91125 \\ ${ }^{b}$ AURA New Initiatives Office, Tucson, AZ \\ ${ }^{c}$ Hertzberg Institute for Astrophysics, Victoria, BC, Canada \\ ${ }^{d}$ University of Chicago, Chicago, IL
}

\begin{abstract}
A parametric model of the dynamic performance of an optical telescope due to wind-buffeting is presented. The model is being developed to support the design of next generation segmented-mirror optical telescopes through enabling rapid design iterations and allowing a more thorough exploration of the design space. A realistic performance assessment requires parametric descriptions of the wind, the structural dynamics, active control of the structure, and the optical response. The current model and its assumptions are presented, with the primary emphasis being on the parameterization of the wind forces. Understanding the temporal spectrum and spatial distribution of wind disturbances inside the telescope enclosure is one of the most challenging aspects in developing the overall parametric model. This involves integrating information from wind tunnel tests, computational fluid dynamics, and measurements at existing observatories. The potential and limitations of control to mitigate the response are also discussed, with realistic constraints on the control bandwidth obtained from the detailed structural model of a particular point design. Finally, initial results are presented on performance trends with a few key parameter variations.
\end{abstract}

Keywords: Parametric modeling, extremely large telescope, control, wind-buffeting

\section{INTRODUCTION}

Various design studies are underway for the next generation of large $(>20 \mathrm{~m})$ ground-based optical telescopes. ${ }^{1,2}$ Several studies have analyzed the wind-induced buffeting of the telescope structure that results from turbulence inside the telescope enclosure ${ }^{3-5}$ and concluded that this vibration must be taken into account in the design process, affecting the design of the telescope enclosure, the telescope structure and the active control system. Estimating the degradation in image quality that results from the wind-induced vibration requires understanding the wind inside the enclosure, coupling these forces to a structural model, estimating the achievable reduction in vibration using active control, and computing the optical consequences of the residual vibration. The key questions that must be answered by this modeling are the extent to which wind-buffeting of the structure is a sufficiently important problem to influence the choice of major telescope design decisions (e.g. focal ratio, elevation-axis location, etc.), and what design options exist to mitigate the problem (increased control bandwidth, larger enclosure, etc.).

Integrated models are being developed to predict the actively controlled structural response at a particular point in the overall design space. ${ }^{6-8}$ Many of the design decisions required in the design of a new telescope must be informed by the predictions of dynamic performance, and thus in order to explore more of the design space, models are required that can rapidly predict performance trends as a function of design parameters. Such a parametric model inevitably requires assumptions, with fidelity sacrificed for the efficiency of exploring the design space. Thus the parametric model is complementary in function to higher fidelity detailed models, with the latter anchoring the predictions of the former at one or more points in the design space. An initial parametric model of wind-buffeting of a telescope was developed by Padin. ${ }^{3}$ The model presented here builds on this earlier work by (i) updating the wind model based on new information, (ii) replacing the structural model by a more 
realistic (but not currently parameterized) particular point design, and (iii) using this point design to more realistically set achievable control bandwidths.

Wind loads are relevant both over the primary mirror (M1) and on the secondary mirror (M2) and nearby supporting structure. Although the cross-sectional area of the latter is small compared to that of M1, the structure around the mirror is exposed to much higher wind loads. Steady forces can be compensated by active control of the telescope structure. Estimates of the unsteady forces can be made from knowledge of the unsteady turbulent wind velocity. The wind parameterization herein reflects our best current understanding of the wind environment inside a telescope enclosure, informed by three separate sources of data. Some limited but valuable data is available from measurements taken at Gemini South observatory. ${ }^{9,10}$ More extensive data under more controlled conditions has been collected in scaled wind-tunnel experiments; while this approach has been used for many years, ${ }^{11}$ modern experimental techniques allow significantly more data to be collected. ${ }^{12,13}$ Finally, computational fluid dynamics $(\mathrm{CFD})^{13-15}$ is an ideal design tool, and can take advantage of the experimental data for validation, so that CFD can be used with confidence in future design work. The parametric model of wind forces herein builds on earlier efforts ${ }^{3,4}$ by incorporating new information from recent computational and wind tunnel studies.

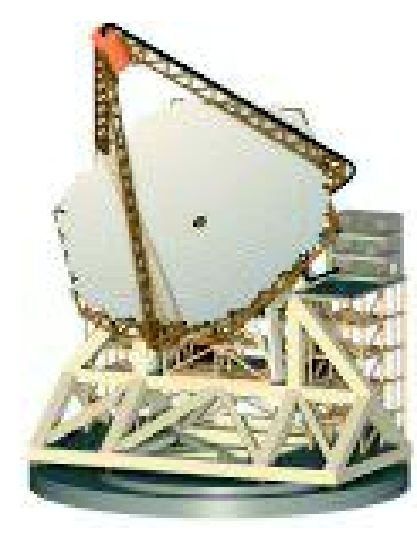

Figure 1. The GSMT f/1 telescope structure, with Cassegrain secondary and elevation axis behind the primary mirror.

The structural model is not yet parameterized; future efforts will include some parameterization of the structural finite element model (FEM) node geometry by using an input file as was done with VLOT. ${ }^{16}$ Performance predictions presented herein use the GSMT $30 \mathrm{~m}$ telescope structural model, ${ }^{17}$ shown in Fig. 1.

Each segment of the primary mirror will have 3 actuators to control its piston, tip and tilt relative to the adjacent segments. For the 36-segment Keck telescopes, the bandwidth of the primary mirror control system is sufficient to compensate only for gravity and thermal disturbances. For future segmented-mirror telescopes, the control bandwidth can be increased so that some of the wind-induced buffeting is compensated. ${ }^{5}$ This is limited by the interaction with lightly damped structural modes. These depend on telescope orientation and therefore it is unlikely that a control algorithm that depends on precise knowledge of them will be sufficiently robust. We use integral control, with the gains optimized as a function of the structural damping to achieve the maximum performance. The secondary mirror will also be actively controlled in 5 degrees of freedom, with higher bandwidth expected on the tip/tilt axes by using reaction mass compensation.

A linear optical model ${ }^{18}$ is used to evaluate the performance. This predicts the optical path difference (OPD) using the first 9 terms in a Zernike basis.

The next section gives an overview of the design parameters included. Each of the elements of the model will be discussed in turn in the subsequent sections, followed by some initial predictions of performance trends with design parameters in Section 7

\section{PARAMETERS}

The design of a new observatory involves the selection of various design parameters. These can be loosely grouped into top level observatory decisions that influence many subsystems and must typically be chosen early in the design process, and subsystem decisions that while less critical to program schedule nonetheless are significant if they lead to economic solutions to what might otherwise present performance problems. Key design decisions for the observatory that are likely to significantly influence the response of the telescope to wind buffeting include:

1. Gregorian or Cassegrain optical configuration.

2. Primary mirror focal ratio

3. Elevation axis location; in front of or behind the primary mirror. 
These parameters affect wind-buffeting both through their impact on the structural design, and through the implicit choice of a minimum size for the telescope enclosure. In terms of their impact on wind-buffeting response, the first two can be grouped into a single parameter specifying the distance between the primary and secondary mirrors.

Some prediction of wind is required early in the design process in order to help inform these decisions. However, to provide meaningful input, it is also essential that we understand the extent to which wind-buffeting can or cannot be mitigated by the choice of other variables; this is the initial focus of this work. The model should therefore be able to predict how the performance varies as a function of the following parameters, in addition to those listed earlier:

- The diameter of the dome (or equivalently, the distance between the secondary mirror and the dome),

- The (passive or active) structural damping,

- The bandwidth of the primary mirror figure control loop, and of secondary mirror position control,

- The control bandwidth of tip/tilt motion, whether achieved through motion of M2 or elsewhere,

- Whether the secondary mirror is adaptive and thus has sufficient figure control bandwidth to compensate for some of the wind-induced deformation of M1,

- The minimum vent area required to obtain adequate dome seeing.

- Structural design variables (e.g. options for coupling the load path from the secondary supporting structure into the main structure),

- External wind speed $U_{\infty}$,

- Telescope azimuth and zenith angle with respect to the external wind direction.

Since the wind is stochastic, the model should ideally predict the fraction of time that the degradation in image quality exceeds some threshold; this therefore requires that the performance be predicted as a function of the external wind speed and viewing angle. Azimuth and zenith angle dependency are not yet included in the model.

The parameterization of venting merits more discussion. While air moving within the enclosure is bad for telescope vibration, some motion is necessary to flush the dome and ensure thermal equilibrium in order to avoid "dome seeing". The telescope enclosure is likely to have vents that can be opened to increase air flow across the primary mirror, particularly in low external wind conditions. How much flushing is required is not yet well understood, and it may be necessary to retain some venting even in high wind conditions when telescope vibration due to wind becomes a problem. Furthermore, for reasons discussed in the next section, some venting may be beneficial in reducing wind-buffeting due to shear layer modes.

\section{WIND}

Wind loads influence the telescope through the forces on the primary mirror, and the forces on the secondary mirror and supporting structure; the latter results in motion of M2 and also deformation of M1 through the structural coupling from the attachment of the supporting structure. In principle, wind loads on the dome could also be transmitted to the structure through the pier, although this effect is believed to be smaller and is currently neglected. The optical consequences of the wind result primarily from the motion and deformation of M1 and M2. The performance is less sensitive to the motions of M3 and the Nasymth platforms. We first discuss the different mechanisms for wind loads on the telescope, followed by the structure of the parameterization, and finally discuss detailed data used to support the selection of wind parameters.

\subsection{Mechanisms}

The structure of the wind flow inside the dome involves several mechanisms. In addition to the mean flow pattern inside the dome, there is broadband turbulence, generated by the flow passing over and through the dome opening, and also generated by the flow entering the dome through vents. Finally, if the dome opening is facing upwind, there may also be significant tones associated with "shear layer modes".

The pressure that results from broadband turbulence inside the telescope enclosure is assumed to have a von Karman temporal power spectrum, $\Phi(f) \propto\left(f_{0}+f\right)^{-7 / 6}$. While the turbulence is clearly not isotropic, this 

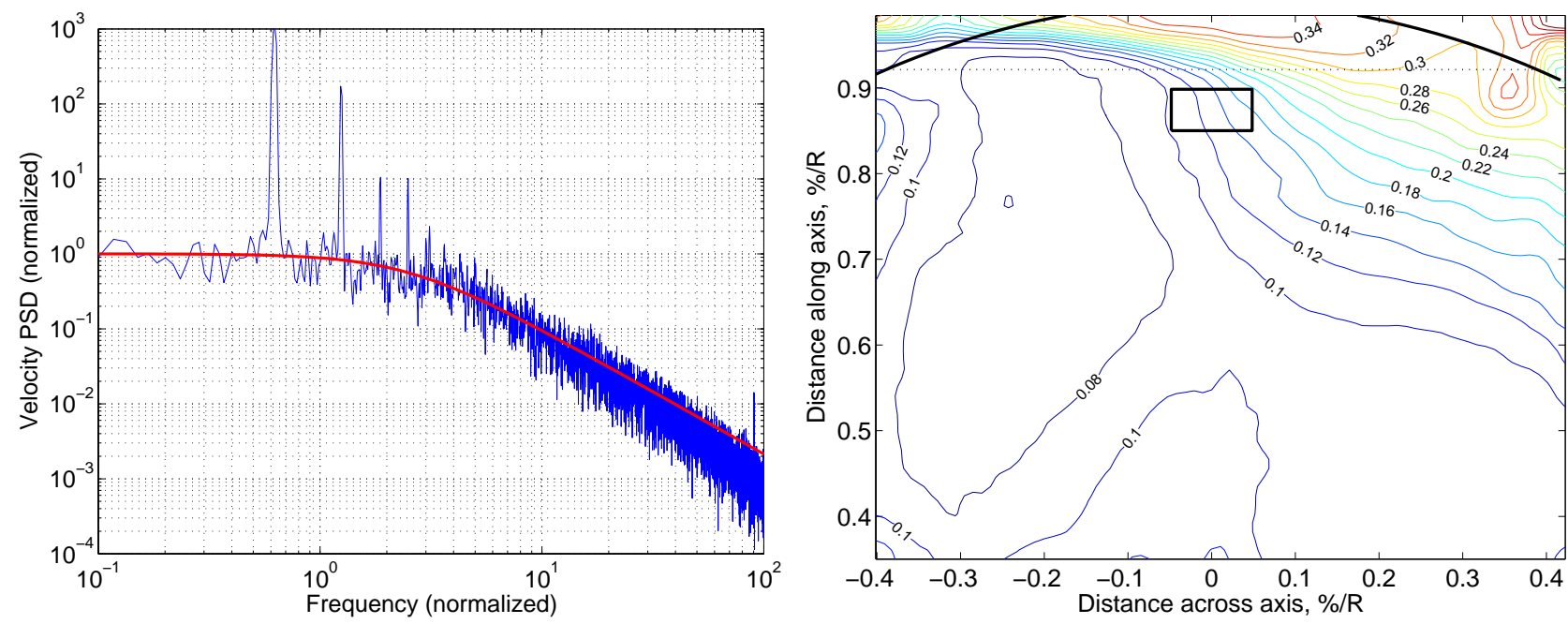

Figure 2. Turbulence characteristics from wind tunnel tests. ${ }^{12}$ Spectrum of turbulence (left) inside a telescope enclosure from hotwire velocity measurement, and comparison with von Karman spectrum $\Phi(f) \propto\left(f_{0}^{2}+f^{2}\right)^{-5 / 6}$. Data is on the optical axis at $\sim 95 \%$ of the radius for dome facing upwind with external wind speed $35 \mathrm{~m} / \mathrm{s}$, the frequency axis is normalized by the external velocity and dome opening diameter. The frequency $f_{0}$ used for comparison includes the reduction in mean wind speed. The first four shear layer modes are clearly visible in the data. Contour plot (right) of the turbulent intensity near the dome opening normalized by the external wind speed, obtained from Digital Particle Image Velocimetry (DPIV) data, with the radius of the dome and a nominal M2 location shown for example. The optical axis in the figure is vertical, the Zenith angle in the test is $30^{\circ}$; the incoming wind is from the upper left. The large turbulence on the right results from the $n=1$ shear layer mode.

assumption is consistent with observations at Gemini ${ }^{5,10}$ as well as measurements in wind tunnel testing (see Fig. 2) and computational results. The outer scale $L_{s}$ associated with the turbulence observed near the secondary mirror, and the turbulence near the primary mirror when the vents are closed is roughly equal to the diameter $D_{s}$ of the dome opening. ${ }^{5,10}$ The outer scale $L_{v}$ associated with the turbulence over M1 that is caused by flow through open vents is related to the height $D_{v}$ of the vent gates. ${ }^{10}$ The spatial correlation length $\ell$ of pressure on a surface resulting from turbulence is related to the outer scale by $\ell=L / \sqrt{70.8} .^{19}$ The spatial correlation must be accounted for in computing forces to apply to the structure; an exponential correlation function $R(r)=e^{-|r| / \ell}$ is used herein as an approximation.

The shear layer modes result from flow passing over an open cavity. ${ }^{20}$ The differential flow speed between the inside and outside flow in the shear layer generates vorticity, which rolls up into large vortices. As these encounter the end of the cavity opening, there is a reflection which propagates upstream to interact with the flow at the initial separation point. This feedback leads to an organization of the shear layer vorticity into modes, with $n$ vortex structures across the length (in the flow direction) of the cavity opening. The oscillation frequency of these modes scales with $n \sigma U_{\infty} / D_{s}$, where $0.5<\sigma<1$ depends on the geometry. These tones occur in wind-tunnel tests at Caltech ${ }^{12}$ and NRC, ${ }^{13}$ and in CFD analyses of these geometries, ${ }^{13,15}$ and for certain configurations are the dominant source of pressure variations within the telescope enclosure. The observed characteristics of these modes include a large oscillatory pressure throughout the entire telescope enclosure, and a large oscillatory vortex structure in the region of the enclosure near the secondary mirror and supporting structure. The scale factor $\sigma$ is approximately 0.65 in all of these cases. However, since it is the local flow velocity across the opening that matters, and not the external wind speed, the factor will depend somewhat on the angle of the dome opening with respect to the wind.

While the frequency of oscillation is straightforward to predict, predicting the amplitude of the tones is further complicated by the potential interaction between these modes and the Helmholtz resonance of the cavity itself. At high external velocities, the $n=1$ shear layer mode may be at a comparable frequency to the Helmholtz resonance and thus dominate the spectra, while at lower velocities, an $n=2$ or $n=3$ mode may become most 
significant. The strength of the shear layer is therefore influenced by how damped the Helmholtz resonance is, which in turn is influenced by how much vent area is open. We capture this effect by multiplying the predicted strength of the shear layer tones in our wind model by a scale factor $\alpha_{v}<1$; the data used to identify this factor will be discussed later. The strength of the shear layer is also influenced by details of the dome near the opening; any external structure will create streamwise vorticity which helps break up the spanwise vorticity rollup responsible for the shear layer modes. This likely explains why the shear layer mode is relatively weak in data collected at Gemini, and only visible at all in the data collected with the vent gates closed. ${ }^{10}$ To account for this effect in the model, we introduce a second scale factor $\alpha_{s}<1$. An accurate assessment of this factor will require detailed modeling with particular dome and shutter designs; until this has been done we assume that $\alpha_{s}=0.25$ will not be difficult to achieve. If realistic shutter configurations, rather than the clean dome geometries used in wind-tunnel and computational efforts, are insufficient alone to achieve this reduction, then it is likely that a relatively modest effort in building dedicated devices to interfere with the shear layer development would suffice.

With vents closed, then the flow observed in wind-tunnel and CFD results is dominated by the shear layer mode. With vents open, the shear layer mode is reduced, but there can be significant broadband turbulence across the primary mirror. In order to explore these effects in the model, this turbulence is scaled by a factor $\beta<1$ that represents the fractional open area of the vents. If dome seeing is not taken into account, then the optimal venting for wind-buffeting effects will result from a trade-off between the reduction in shear layer tone amplitude and the increased broadband forcing across the primary mirror.

One final issue to consider is that it is the pressure difference between the front and back side of the primary mirror that influences the motion of the mirror. For the broadband turbulence, the correlation between pressure on the upper and lower surfaces of the primary mirror is not known. However, since the correlation lengths are short compared to the mirror diameter, it is unlikely that significant correlation exists. Structure behind the mirror is likely to reduce flow speeds and hence turbulent wind forces, hence it is reasonable to ignore the broadband forces on the back of the mirror. The shear mode, however, excites M1 through pressure waves propagating from the dome opening, with wavelength much longer than the size of the mirror. Thus the back of the mirror may see similar pressure to the front, significantly reducing the net effect on M1 of these forces. We currently do not have data or analysis to estimate the net reduction, so for the purposes of initial modeling, we conservatively assume that the back side of the mirror is not exposed to any wind loads.

\subsection{Parameterization:}

The wind on the primary mirror is broken down into the three components discussed above;

1. The shear layer mode,

2. Broadband turbulence generated by flow across the dome opening,

3. Broadband turbulence generated by flow passing through the vents.

The pressure forces for all of these components are assumed to scale with the external dynamic pressure $\left(\frac{1}{2} \rho U^{2}\right)$.

The distributed wind pressure over a surface can be simulated with a finite number of forces represented by the vector $f \in \mathbb{R}^{N}$ at $N$ points $x_{i}$ driven by $N$ independent noise sources $w \in \mathbb{R}^{N}$. The correct correlation $R_{i j}$ between pressures at locations $x_{i}$ and $x_{j}$ is obtained with $f=\Gamma w$ and $\Gamma \Gamma^{T}=R$. With $A_{i}=\pi(D / 2)^{2} / N$, then

$$
f_{\mathrm{M} 1}=\left(\frac{1}{2} \rho U^{2}\right) A_{i}\left(a_{1} \Gamma_{1} w_{1}+a_{2} \Gamma_{2} w_{2}+a_{3} \Gamma_{3} w_{3}\right)
$$

where

$$
\begin{array}{ccc}
\Gamma_{1} \in \mathbb{R}^{N \times N}=R_{1}^{1 / 2} \quad\left(R_{1}\right)_{i j}=e^{-\left|x_{i}-x_{j}\right| / \ell_{1}} & \ell_{1}=\frac{D_{s}}{\sqrt{70.8}} \\
w_{1} \in \mathbb{R}^{N} \text { uncorrelated with PSD } \Phi_{11}=\left(f_{1}^{2}+f^{2}\right)^{-7 / 6} & f_{1}=D_{s} / U_{1 s} \\
\Gamma_{2} \in \mathbb{R}^{N \times N}=R_{2}^{1 / 2} \quad\left(R_{2}\right)_{i j}=e^{-\left|x_{i}-x_{j}\right| / \ell_{2}} & \ell_{2}=\frac{D_{v}}{\sqrt{70.8}}
\end{array}
$$




$$
\begin{gathered}
w_{2} \in \mathbb{R}^{N} \text { uncorrelated with PSD } \Phi_{22}=\left(f_{2}^{2}+f^{2}\right)^{-7 / 6} \quad f_{2}=D_{v} / U_{1 v} \\
\Gamma_{3} \in \mathbb{R}^{N}, \quad \Gamma_{3}=\left[\begin{array}{llll}
1 & 1 & \cdots & 1
\end{array}\right]^{T} \quad w_{3}=\sin \left(f_{\mathrm{sh}} t\right) \quad f_{\mathrm{sh}}=0.65 n U_{\infty} / D_{s}
\end{gathered}
$$

The wind force on the secondary mirror and supporting structure is broken down into two components,

1. The shear layer mode

2. Broadband turbulence generated by flow passing through the dome opening,

with forces in both axial and transverse directions. The distributed forces across the support structure are lumped into an equivalent force on M2 itself.

The transverse force on the secondary mirror and supporting structure results both in deflection of M2 and deformation of M1 through the resulting torque. Assuming an exponential correlation function and roughly equal rms pressure acting across the structure that supports the secondary mirror (see Fig. 3), then the equivalent force on M2 that yields the same net torque on M1 can be derived from integration. For correlation length $\ell$ and distance between $\mathrm{M} 1$ and $\mathrm{M} 2$ of $H$, the result is to include an effective area based on the effective length $d_{\text {eff }}=\sqrt{2 \ell H / 3-\ell^{2}}$ and the cross-sectional width $h$ of the supporting structure.

$$
f_{\mathrm{M} 2} \in \mathbb{R}^{2}=\left(\frac{1}{2} \rho U_{\infty}^{2}\right)\left(\left(a_{4 a} A_{2}+a_{4 b} A_{\text {eff }}\right) w_{4}+\left(a_{5 a} A_{2}+a_{5 b} A_{\text {eff }}\right) w_{3}\right)
$$

where $w_{3}$ is the same shear layer disturbance that affects the primary mirror, and $w_{4} \in \mathbb{R}^{2}$ is uncorrelated with $\operatorname{PSD} \Phi_{44}=\left(f_{4}^{2}+f^{2}\right)^{-7 / 6}, f_{4}=D_{s} / U_{2}$

\subsection{Parameter estimates}

Several of the parameters in the equations from the previous subsection have already been discussed, such as $\alpha_{s}$ and $\beta$ and the relationship between the outer scale and the source of turbulence.

Estimates of the pressure on the surface of the primary mirror are derived from measurements collected at Gemini observatory, with additional information available from wind-tunnel and computational models conducted by IAR at NRC for the VLOT telescope design. ${ }^{13}$ Developing estimates of the forces acting on the secondary mirror and supporting structure is complicated by the desire to predict these forces as a function of the location of the mirror within the dome (i.e. to estimate the benefit from building a slightly larger dome with greater clearance between the secondary mirror and the shear layer). Thus it is insufficient to test a particular design with M2 at a particular location. Measurements of the mean and turbulent wind velocity inside an empty dome have been taken in a wind tunnel model at Caltech ${ }^{12}$ and matching computations conducted at AURA. ${ }^{15}$ The pressure force can be estimated from the velocity if one assumes that the structure would not have significantly altered the flow pattern. This would clearly be a poor assumption for computing the forces on the primary mirror, but the cross-sectional area of the secondary mirror is relatively small compared to the open area of the dome (roughly 1\%). The velocity can be separated into mean and fluctuation components as $u=\bar{u}+u^{\prime}$. The pressure due to drag is $p=C_{d}\left(\frac{1}{2} \rho u^{2}\right)=C_{d} \frac{1}{2} \rho\left(\bar{u}^{2}+2 \bar{u} u^{\prime}+u^{\prime 2}\right)$. The steady pressure from the first term is not of interest, since it can be compensated by the telescope active control system. Near M2, the mean velocity in the transverse direction is much larger than the fluctuation component, so that $p \simeq C_{d} \frac{1}{2} \rho 2 \bar{u} u^{\prime}$. The mean velocity in the axial direction is much smaller than the fluctuation component, so that $p \simeq C_{d} \frac{1}{2} \rho\left(u^{\prime}\right)^{2}$. For high Reynolds numbers, the drag coefficient for a flat plate is approximately one, while that for a cylinder in a cross-flow is closer to $1 / 2$. The secondary mirror structure is assumed to be cylindrical. The supporting legs for the structure are assumed to be rectangular, with negligible cross-sectional area in the axial direction in order to minimize light blockage of the primary mirror. Note that this computation ignores von Karman shedding from the structural elements, which may also be relevant.

The frequency of the shear layer mode is set to $0.65 n U_{\infty} / D_{s}$. Based on wind-tunnel testing with even small amounts of venting to damp the effects of the Helmholtz mode, the $n=2$ mode is typically dominant. For a $30 \mathrm{~m}$ dome opening in a $12 \mathrm{~m} / \mathrm{s}$ external wind, this leads to a frequency of roughly $0.5 \mathrm{~Hz}$; well below the first structural modes of the telescope, but at a high enough frequency where the active control is likely to achieve only modest reductions. The acoustic wavelength at this frequency is approximately $660 \mathrm{~m}$, and thus it is reasonable 
to assume that the pressure field across the primary is uniform as indicated by $\Gamma_{3}$ in Section 3.2; this is consistent with observed data from the NRC wind-tunnel test. The forces on the secondary and supporting structure are nearly in phase with the forces on the primary, but much larger pressures are possible due to the drag forces that result from the large oscillatory velocities from the vortex structures of the shear layer modes.

The amplitudes $a_{1}$ through $a_{5}$ for an upwind telescope orientation are obtained as follows:

- $a_{1}$ (broadband on M1 due to dome opening): Measurements taken at Gemini observatory with the vents closed and the telescope facing upwind gave that on average the rms velocity at the primary mirror was $6 \%$ of the external wind speed, ${ }^{5}$ and that this was a good predictor of both the rms pressure on the primary mirror and the outer scale frequency $f_{1}=D_{s} / U_{1 s}$. The internal wind speeds are assumed to scale with the ratio of dome opening size to dome diameter, ${ }^{3}\left(D_{s} / D_{d}\right)^{2 / 3}$. Data collected in the NRC wind tunnel test suggest that the rms pressures may be somewhat higher, but this data has not yet been fully post-processed to separate the effects of the broadband turbulence from the shear layer.

- $a_{2}$ (broadband on M1 due to vents): Measurements taken at Gemini observatory with the vents fully open gave that on average the rms velocity at the primary mirror was $30 \%$ of the external wind speed. ${ }^{10}$ We assume that the average velocity scales with the fractional open area of the vents. This estimate will be updated with additional measurements from the NRC wind tunnel tests.

- $a_{3}$ (shear layer on M1): The peak-to-peak pressure amplitude for the shear layer mode on the primary can be estimated from theory ${ }^{15}$ for deep cavities as no larger than $p=(1 / 3)\left(\frac{1}{2} \rho U^{2}\right)$. This is consistent with the pressure levels observed in CFD for two different dome geometries without venting.

- $a_{4}$ (broadband on M2): The DPIV data collected in the Caltech wind tunnel test ${ }^{12}$ in the region near the dome opening with vents open is dominated by the broadband turbulence, rather than the shear layer mode (see Fig. 3). The axial forces on M2 due to drag result from the turbulent velocity $\left(v^{\prime}\right)^{2}$ while the transverse forces result from $2 \bar{u} u^{\prime}$ as described earlier. A simple fit of the data along the telescope optical axis is compared with the DPIV data in Fig. 3(b).

- $a_{5}$ (shear layer on M2): The DPIV data collected in the Caltech wind tunnel test in the region near the dome opening without vents contains significant energy associated with the shear layer mode, rather than solely broadband turbulence (see Fig. 3(a)). The data along the telescope axis are fit as described above (Fig. 3(c)), and the mean-square values associated with the shear layer mode obtained by subtracting the mean-square values associated with the broadband turbulence.

In addition to the amplitude scalings, the values of several other parameters can be estimated. The dependence of the amplitude of the shear layer mode on the open area of venting (relative to the open area of the dome) is shown in Figure 3 for hot-wire measurements of velocity taken in the Caltech wind tunnel test. The total mean-square velocity in the first 3 shear layer modes was computed, normalized by the total mean-square velocity in the broadband turbulence. Even modest amounts of venting reduces the shear layer amplitudes significantly; achieving $\alpha_{v}=0.25$ is plausible for a $10 \%$ open area. If the total venting area is roughly half the open area of the viewing opening, then choosing $\beta=0.25$ is roughly consistent with choosing $\alpha_{v}=0.25$.

At present, we have not yet estimated the dependency of all of the amplitudes with azimuth angle and zenith angle. The shear layer modes are not present when facing downwind as the dome opening will be in a separated flow regime (for azimuth angles greater than about 100 degrees). However, the broadband turbulence near M2 may actually increase in downwind orientations. ${ }^{12}$

\section{STRUCTURE}

The structural model needs to be sufficiently detailed to predict the first few modes accurately, and predict higher frequency modes with sufficient accuracy to capture the resulting impact on achievable control bandwidth.

Finite element models being developed for possible structural designs of TMT will use a parameterized geometry file that can make predictions of structural dynamic changes due to design parameter variations relatively rapid. The FEM software can also re-optimize structural variables after changing parameters. Changes in elevation axis would require a significantly different structural design and cannot be easily parameterized. The model 

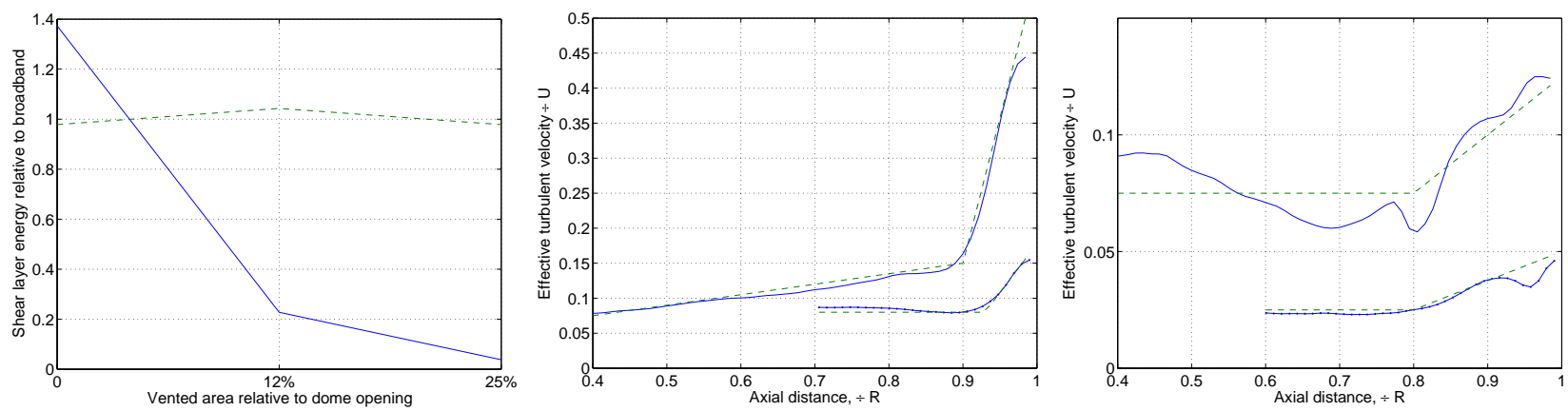

Figure 3. Wind-tunnel measurements used for defining parameters. The left plot shows the energy in first three shear layer modes (solid) normalized by the broadband turbulent energy as a function of the venting area open (relative to the open area of the dome), obtained from hotwire measurements of velocity in the shear layer at $19 \mathrm{~m} / \mathrm{s}$ external wind speed. The middle and rightmost plots show the effective velocity used for computation of transverse and vertical pressure forces respectively on the secondary mirror as a function of M2 location, from DPIV data (solid) and fit (dashed). In each case, the upper pair of curves correspond to data with the vents closed (dominated by shear layer modes) while the lower pair are with vents open ( $25 \%$ area of dome opening, flow dominated by broadband turbulence).

described herein does not yet include variation of structural design parameters other than the damping ratio, as we have chosen to focus our current efforts on the wind parameterization. Predictions shown in Section 7 use the GSMT structure. ${ }^{17}$

The first 100 structural modes of the GSMT FEM have been retained for analysis, with the first flexible mode is at $2.2 \mathrm{~Hz}$. Although the FEM does not have segment-level spatial resolution of the primary mirror, this is not a limitation for predictions of the seeing-limited consequences of wind-buffeting. Including the finite stiffness of individual actuators and whiffle trees on the back of each segment could be done, ${ }^{6}$ however, the seeing-limited image degradation is dominated by global (low wave number) deformation of the primary mirror which is dominated by the compliance of the mirror cell, not the actuator and whiffle tree compliance. Currently only nine terms in the Zernike expansion of primary mirror deformation are retained in the predictions of optical consequences. The GSMT FEM includes nodes corresponding to 91 raft centers (a raft consists of 7 segments) which is sufficient to estimate these 9 terms.

\section{CONTROL}

Since both the primary and secondary mirror must have a control system to compensate for gravitational deflections, increasing the control bandwidth to the maximum achievable may be the most economical mitigation strategy if it is determined that wind-buffeting is a problem. For the secondary mirror, control of rigid body position is essential, but control of figure degrees of freedom at a sufficient bandwidth to mitigate wind-induced performance problems will not be feasible without an adaptive secondary mirror. Thus an additional option to consider in future analysis is the extent to which such a mirror could compensate for wind-induced primary mirror figure errors and improve performance.

The key parameters associated with the control are the bandwidths of the primary mirror figure correction, the secondary mirror position and tip/tilt control, and the bandwidth (high or low with respect to the wind) of the secondary mirror figure control. Initial parametric modeling included a simple high-pass filter for the effect of the control. ${ }^{3}$ This can be improved by incorporating the dependency of achievable control bandwidth on the design parameters, and also by including a more realistic sensitivity function that correctly accounts for amplification above the control bandwidth. The bandwidth is limited by interaction with structural modes, and thus depends on both the frequency and the damping in the structure (which could be achieved passively or perhaps actively).

The structural modes vary with the telescope elevation angle, and are unlikely to be known with sufficient accuracy for detailed information about them to be reliable for control design. Therefore we assume a simple controller structure that does not depend on details of the structure, and which will therefore be robust with 

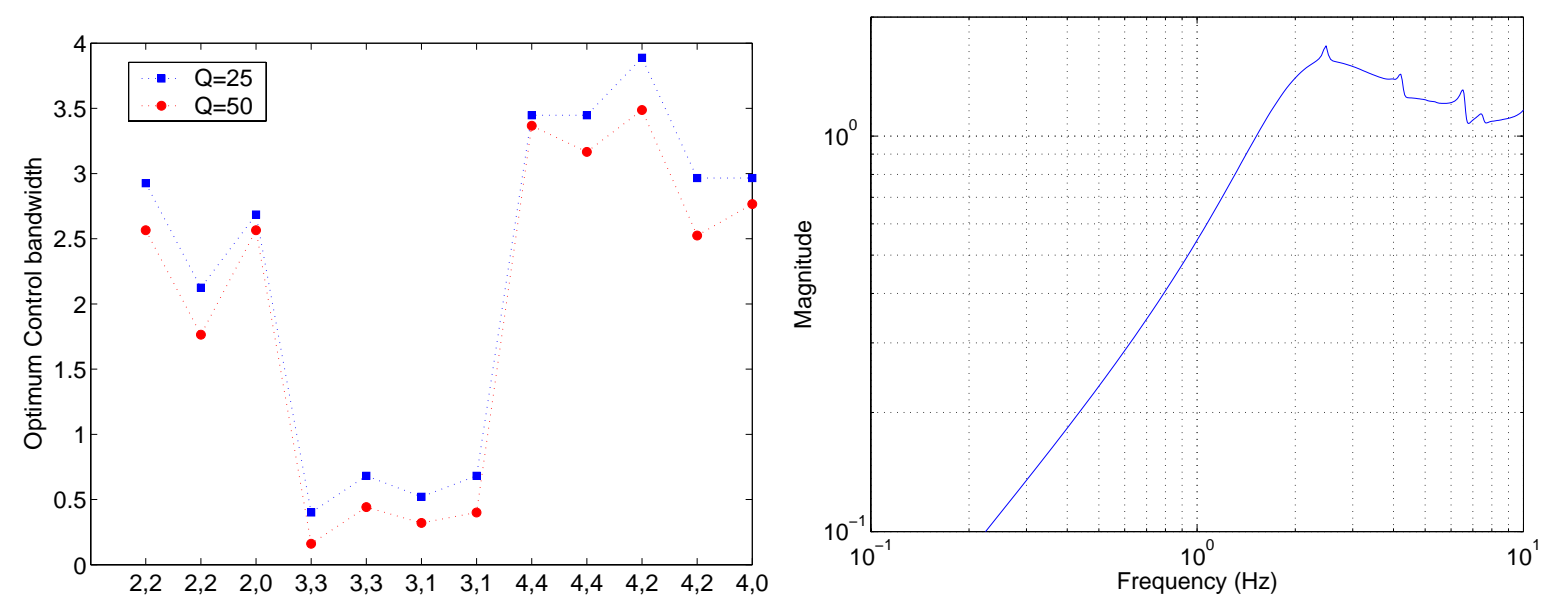

Figure 4. Control of GSMT primary mirror. Optimal control bandwidth (left) for different Zernike basis functions of M1 deflection as a function of structural damping $Q$. A typical sensitivity function is shown on the right for one of the astigmatism modes and $Q=50$.

respect to variations and uncertainty. An integral controller is used, with an additional low pass filter above the bandwidth to gain-stabilize higher frequency modes. (While collocated transfer functions are strictly positive real, phase stabilization seems implausible without excessively high sample rates on the control to minimize the time delay.) Thus we assume control laws of the form

$$
K(s)=G(0)^{-1} \frac{\omega_{c}}{s} \cdot \frac{1}{s / \omega_{r}+1}
$$

where $G(0)$ is the static gain of the transfer function being controlled, and the parameters $\omega_{c}$ and $\omega_{r}$ are optimized for each relevant degree of freedom.

Segmented-mirror telescopes are presumed to have edge-sensors similar to Keck, which can sense all of the degrees of freedom of the primary mirror other than piston, tip and tilt. ${ }^{21}$ The contribution to surface errors due to sensor noise is well understood ${ }^{22}$ and can be separately added. Therefore for the purposes of this model, we can presume that the deflections other than global piston, tip and tilt are directly measured. Typical control development for segmented-mirror telescopes assumes that the control for each actuator is the same. However, the lowest frequency structural modes involve "global" deformation of the structure while higher frequency modes involve primarily higher wave number deformations. If the same bandwidth applies to all the degrees of freedom, then that bandwidth will be limited by interaction with the lowest structural modes. An alternative is to project the global motion out of the observed degrees of freedom, develop a separate controller for this motion, and add the result back into the individual actuator commands. Since the structural mode shapes are not likely to be sufficiently well known to be used as a basis set for describing global motion, we use a Zernike basis $\Phi_{G}$. Thus the final actuator command would be of the form $u=K_{L}\left(x-\Phi_{G}^{T} x\right)+\Phi_{G} K_{G}\left(\Phi_{G}^{T} x\right)$ where $K_{G}$ is designed for the first few Zernike basis functions, and the "local" control $K_{L}$ compensates for the higher order deformations, at a higher control bandwidth than would otherwise be achievable.

The surface deflection of the primary mirror is therefore transformed into deflections of each Zernike basis function, the control for each of these assumed of the form of Eq. (3), and the parameters optimized to minimize performance for the assumed wind loads from Section 3. The optimal bandwidth as a function of mode number for two different values of the structural damping is shown in Fig. 4. For this particular structure, the modes with odd radial degree couple strongly into the first few modes of the entire telescope structure and are therefore limited in bandwidth by these modes. The modes with even radial degree are limited in bandwidth only by higher frequency modes of the mirror cell, and can be controlled with much higher bandwidth.

The structural deflections obtained from applying the wind forces to the structure are filtered by the sensitivity function corresponding to the optimized controller. A typical sensitivity function (for one of the astigmatism degrees of freedom of the primary mirror) is shown in Fig. 4. 


\begin{tabular}{|c|l|rl|}
\hline Parameter & Description & Value & \\
\hline$D$ & M1 diameter & 30 & $\mathrm{~m}$ \\
$D_{s}$ & Dome opening width & $1.1 D$ & \\
$D_{d}$ & Dome diameter $(2 R)$ & 75 & $\mathrm{~m}$ \\
$H$ & Distance M1 to M2 & $0.9 D$ & \\
$U_{\infty}$ & External wind speed & 12 & $\mathrm{~m} / \mathrm{s}$ \\
& M2 location & $0.85 R$ & \\
& M2 diameter & 3.5 & $\mathrm{~m}$ \\
$h$ & M2 height & 2 & $\mathrm{~m}$ \\
$D_{v}$ & M2 support structure width & 0.5 & $\mathrm{~m}$ \\
$\rho$ & vent height & 10 & $\mathrm{~m}$ \\
& atmospheric density & 0.819 & $\mathrm{~kg} \mathrm{~m}{ }^{3} / \mathrm{s}$ \\
$Q$ & tip/tilt bandwidth & 10 & $\mathrm{~Hz}$ \\
$\alpha_{s}$ & Structural damping & 50 & \\
$\alpha_{v}$ & Shear layer reduction from dome & 0.25 & \\
$\beta$ & Shear layer reduction from vents & 0.25 & \\
\hline
\end{tabular}

Table 1. Nominal parameters used in simulation

The secondary mirror will be used for chopping, and to accomplish this, the actuation of the tip/tilt degrees of freedom must be compensated by a reaction mass that minimizes the excitation of the structural modes. An evaluation of the achievable bandwidth of such a system requires an assumption on the accuracy to which the reaction mass can be designed. For the purposes of the subsequent analysis, we assume a $10 \mathrm{~Hz}$ closed loop control bandwidth, and use a simplified high-pass filter for the corresponding sensitivity function for these degrees of freedom. Once the tip/tilt of the primary and secondary mirrors is compensated, the residual tip/tilt of the image is dominated by the residual decenter of M2 (above the achievable bandwidth for this degree of freedom). Compensating for this motion using the tip/tilt of the secondary introduces coma errors that can partially be corrected with a corresponding command to the primary mirror control system, albeit at a lower bandwidth. This additional coma error is not yet included in the simulation results presented in Section 7 .

\section{IMAGE QUALITY}

The optical consequences can be reasonably approximated via a linear optical model, already available as noted earlier. ${ }^{18}$ We currently include only the effect of 9 Zernike displacements of M1 and 5 degrees of freedom of M2 motion. Based on this, we compute two scalar metrics for seeing-limited observations; the rms image motion (tip/tilt) and the rms of the tip-tilt-removed OPD.

\section{PREDICTIONS}

The components of the model discussed in the previous 4 sections can be used to predict the OPD resulting from the controlled response of the structure due to the wind loads. This computation is done in the frequency domain. The nominal values for all parameters used are shown in Table 1. The parameters correspond to the telescope facing upwind in a high wind case $\left(85^{\text {th }}\right.$ percentile on Mauna Kea) and thus represent close to a worstcase situation. Furthermore, since the structural design of GSMT was not optimized with respect to the wind disturbance, the absolute magnitude of these results should be interpreted cautiously.

For the parameters listed in Table 1, the wind loads were applied to the GSMT structure, and the optical response computed with and without control. The OPD is separated into the tip/tilt and higher order components, and the rms value computed. The results are shown in Figure 5 as a function of frequency, and as a function of the source mechanism. The first peak in the spectrum is due to the shear layer mode, the second peak results from the response of the first structural mode. For the parameters chosen, the broadband turbulence acting on $\mathrm{M} 2$ is the dominant contribution to $\mathrm{OPD}$, however, none of the components are negligible. 

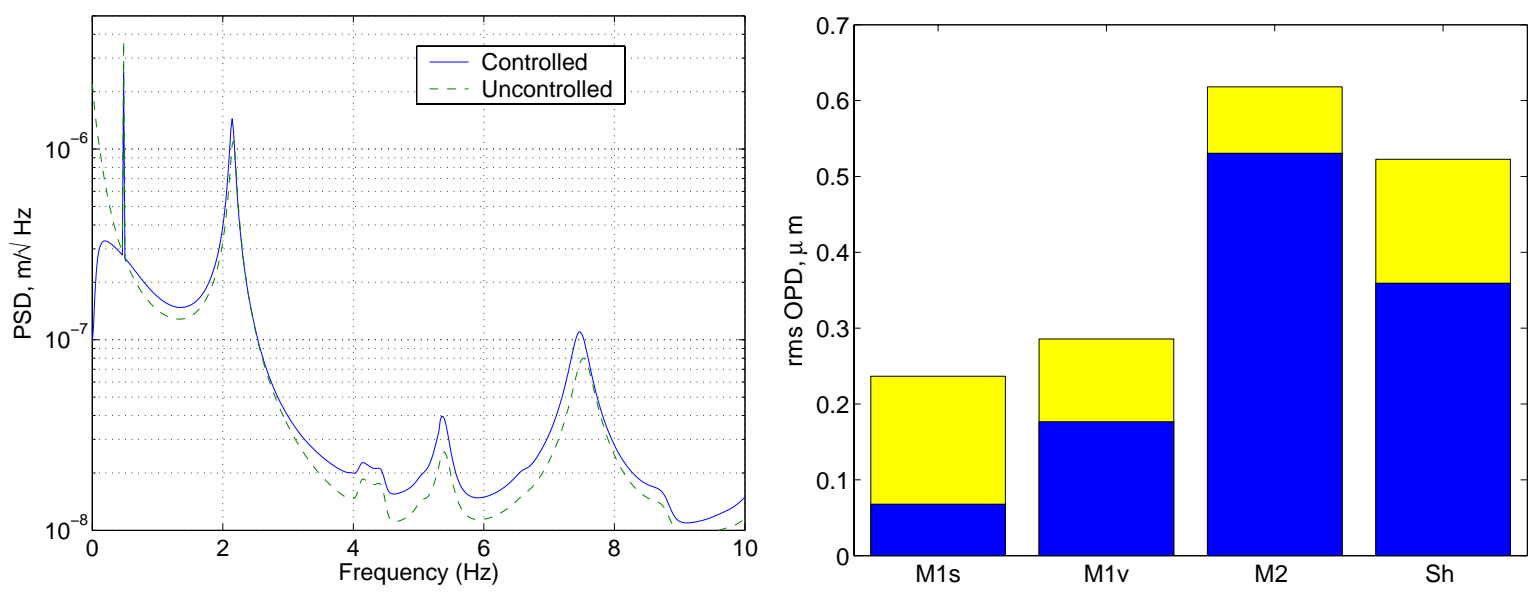

Figure 5. rms OPD (tip/tilt removed) for nominal set of parameters, dependence on frequency (left), uncontrolled and controlled, and dependence on the wind mechanism (right, solid bars indicate controlled response). Wind mechanisms are broadband on M1 due to dome opening and vents, broadband forces on M2, and shear layer.
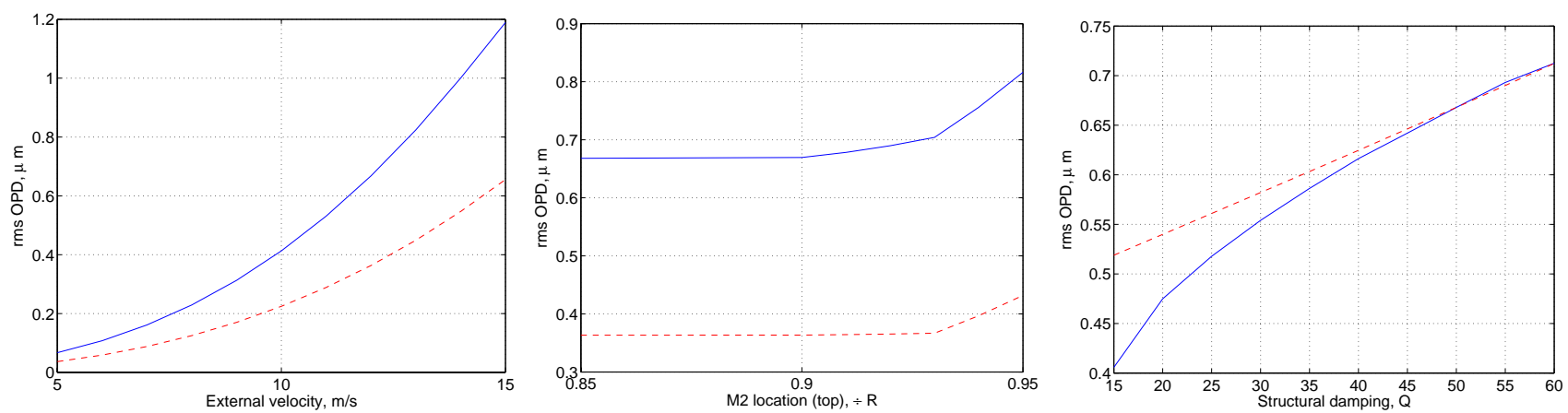

Figure 6. Variation in closed-loop rms OPD with tip/tilt removed (solid) and tip/tilt (dashed) as a function of external velocity (left) and the location of the top of the M2 structure within the dome (middle). Variation as a function of structural damping (right), with (solid) and without (dashed) the control bandwidth re-optimized.

With all the other parameters held constant, the variation in the same performance metric was determined as a function of the incoming velocity, the location of the secondary mirror system, and the structural damping. These plots are shown in Fig. 6. The dependence on velocity is stronger than quadratic because both the frequency of the shear layer mode and the temporal corner frequency of the broadband turbulence components scale with the external velocity. The higher frequency content results in higher relative excitation of the structural modes, and less reduction due to control. The dependence on the location of M2 is negligible once the entire M2 structure is far enough inside the telescope enclosure to be below the shear layer; this result is consistent with the parameterization of wind forces near the secondary shown in Fig. 3.

The dependence of performance on structural damping has been investigated in two ways. First, with fixed control bandwidth (optimized for $Q=50$ ), doubling the damping (to $Q=25$ ) results in an $18 \%$ reduction in rms OPD. Increased damping also permits increased control bandwidth; if the control bandwidth is re-optimized, then $Q=25$ yields a $22 \%$ improvement in OPD.

\section{Acknowledgements}

This work was conducted as part of the Thirty Meter Telescope project. The Thirty Meter Telescope (TMT) Project is a partnership of the Association of Universities for Research in Astronomy (AURA), the Association of Canadian Universities for Research in Astronomy (ACURA), the California Institute of Technology and the University of California. The partners gratefully acknowledge the support of the Gordon and Betty Moore 
Foundation, the US National Science Foundation, the National Research Council of Canada, the Natural Sciences and Engineering Research Council of Canada, and the Gemini Partnership.

The comments of Jerry Nelson at UCSC have been extremely valuable in this effort. Tait Pottebaum at Caltech provided insight into the measured flow patterns.

\section{REFERENCES}

1. Nelson, J. E., "TMT Status," SPIE 5489-27, 2004.

2. Johns, M., Angel, R., Bernstein, R., Fabricant, D., McCarthy, P., Phillips, M., and Shectman, S., "Status of the Giant Magellan Telescope (GMT) project," SPIE 5489-28, 2004.

3. Padin, S. and Davison, W., "Model of image degradation due to wind buffeting on an extremely large telescope," Applied Optics, Vol. 43, No. 3, pp. 592-600, Jan. 2004.

4. Angeli, G., Upton, R., Segurson, A., and Ellerbroek, B., "Active optics challenges for a thirty meter segmented mirror telescope," SPIE 2 ${ }^{\text {nd }}$ Backaskog Workshop on Extremely Large Telescopes, 2003.

5. MacMartin, D. G., "Control Challenges for Extremely Large Telescopes," Proc. SPIE 5054, 2003.

6. Angeli, G., Segurson, A., Upton, R., Gregory, B., and Cho, M., "Integrated modeling tools for large ground based optical telescopes," Proceedings of the SPIE, Vol. 5178, 2003, pp. 49-63.

7. Angeli, G. Z., Roberts, S., MacMynowski, D. G., Fitzsimmons, J., Dunn, J., Vogiatzis, K., and Sheinis, A., "Modeling tools to estimate the performance of the Thirty Meter Telescope: an integrated approach," SPIE 5497-24, 2004.

8. Roberts, S., Pazder, J., Fitzimmons, J., Herriot, G., Loewen, N., Smith, M., Dunn, J., and Saddlemyer, L., "Integrated modeling of the Canadian Very Large Optical Telescope," SPIE $2^{\text {nd }}$ Backaskog Workshop on Extremely Large Telescopes, 2003.

9. Cho, M. K., Stepp, L. M., Angeli, G. Z., and Smith, D. R., "Wind loading of large telescopes," Large Ground-Based Telescopes (Oschmann, and Stepp, eds.), 2002, pp. 352-367. Proc. SPIE 4837.

10. Angeli, G. Z., Cho, M. K., Sheehan, M., and Stepp, L. M., "Characterization of Wind Loading of Telescopes," Proc. SPIE Vol. 4757; Workshop on Integrated Modeling of Telescopes (Andersen, T., ed.), 2002, pp. 72-83.

11. Kiceniuk, T. and Potter, K., "Internal air flow patterns for the Keck 10 meter telescope observatory dome," 1986. Keck Observatory Report \# 166.

12. Pottebaum, T. and MacMynowski, D. G., "Wind tunnel testing of a generic telescope enclosure," SPIE 5495-30, 2004.

13. Fitzsimmons, J., Dunn, J., Herriot, G., Jolissaint, L., Roberts, S., Mamou, M., and Cooper, K., "Predicting the aerodynamic performance of the Canadian Very Large Optical Telescope," SPIE 5497-31, 2004.

14. DeYoung, D. S., "Numerical Simulations of Airflow in Telescope Enclosures," The Astronomical Journal, Vol. 112, No. 6, pp. 2896-2908, Dec. 1996.

15. Vogiatzis, K., Segurson, A., and Angeli, G. Z., "Estimating the effect of wind loading on Extremely Large Telescope performance using Computational Fluid Dynamics," SPIE 5497-30, 2004.

16. VLOT Project Book, Canadian VLOT Working Group, 2003. http://www.hia-iha.nrc-cnrc.gc.ca/VLOT/index.html.

17. Enabling a Giant Segmented Mirror Telescope for the Astronomical Community, AURA New Initiatives Office, 2002. http://www aura-nio.edu/book/index.html.

18. Angeli, G. and Gregory, B., "Linear Optical model for a large ground based telescope," Proceedings of the SPIE, Vol. 5178, 2003, pp. 64-73.

19. Simiu, E. and Scanlan, R. H., Wind Effects on Structures - An Introduction to Wind Engineering, John Wiley \& Sons Inc., New York, 1986.

20. Rossiter, J. E., "Wind-tunnel experiments on the flow over rectangular cavities at subsonic and transonic speeds." Aeronautical Research Council Reports and Memoranda, No. 3438, 1964.

21. Chanan, G., MacMartin, D. G., Nelson, J., and Mast, T., "Control and Alignment of Segmented-Mirror Telescopes: Matrices, Modes, and Error Propagation," Applied Optics, Vol. 43, No. 6, pp. 1223-1232, 2004.

22. MacMartin, D. G. and Chanan, G., "Measurement accuracy in control of segmented-mirror telescopes," Applied Optics, Vol. 43, No. 3, pp. 608-615, 2004. 